\title{
An Improved GM $(1,1)$ Model on Initial Value and Its Application on Power Load Forecasting
}

\author{
Shuangchen Li, Tiantian Wang
}

Department of Economic Management ,North China Electric Power University, Baoding ,Hebei, China

Keyword: GM $(1,1)$ model ; Power load forecasting; residual sum of squares; Initial value.

\begin{abstract}
When Calculating the parameter c of differential equations,traditional GM(1,1) model considers only the of 1-AGO,the oldest history data ,resulting loss of new information to some extent.This paper gives a new way to calculate the initial value.The new model firstly establishes the function of residual sum of squares.Then it solves the equation and gives expression of parameter c. Finally,it uses the new 'c' and obtains the new formula of Predictive value.Through the example analysis,this paper proves that improved prediction model can improve prediction precision.
\end{abstract}

\section{INSTRUCTIONS}

Power load forecasting is the basis of power system planning and an important indicator to measure the modernization of power system management. The essence of power load forecasting is the forecast of electricity market demand.Forecast of future electricity demand can guide the position of power supply and power generation plan in a city.Grey system that with partial information known and partial information unknown is used as the research subject of Grey prediction model .It uses potential rules such as data collection to establish grey model. It uses known information to establish differential equation and then calculate unknown data.The model requires that the original data are non-negative and data series are in line or substantially in line with exponential[Ren Gonghang,2010].

\section{TRADITIONAL GM (1,1) MODEL}

\subsection{Model Introduction}

GM(1,1)model is one of the most commonly used grey model.The concrete process is as follows[Deng Julong,2000][Wang Changjiang,2006][Contreras J, 2003]:

(1)Establish original data sequence $X^{(0)}$

$$
x^{(0)}=\left\{x^{(0)}(1), x^{(0)}(2), \ldots, x^{(0)}(n)\right\}
$$

(2)Establish accumulated generating sequence $X^{(1)}$

$$
\begin{gathered}
x^{(1)}=\left\{x^{(1)}(1), x^{(1)}(2), \ldots, x^{(1)}(n)\right\} \\
x^{(1)}(t)=\sum_{i=1}^{t} x^{(0)}(i)
\end{gathered}
$$

(3)Establish original form and differential equation of $\mathrm{GM}(1,1)$

The original form of $\mathrm{GM}(1,1)$ is as follows:

$$
x^{(0)}(t)+a x^{(1)}(t)=u
$$

Where $a$ is development coefficient, which reflects development trend of data sequence; $u$ is coordination coefficient, which reflects change relationship between data.

Establish the first order differential equation:

$$
x^{(1)}(t)=\frac{u}{a}+c e^{a t}
$$

That is 
$\frac{d x^{(1)}(t)}{d t}+a x^{(1)}(t)=u$

$\frac{d x^{(1)}(t)}{d t}=x^{(0)}(k+1)$

Where, $x$ is the mean value of $k$ and $k+1$.The above equation can be written in matrix form:

$Y_{n}=B \hat{A}$

Where

$$
\begin{aligned}
& Y_{n}=\left[\begin{array}{c}
x^{(0)}(2) \\
x^{(0)}(3) \\
\ldots \\
x^{(0)}(n)
\end{array}\right] \\
& A=\left[\begin{array}{l}
a \\
u
\end{array}\right]
\end{aligned}
$$

$B=\left[\begin{array}{cc}-\frac{1}{2}\left[x^{(1)}(1)+x^{(1)}(2)\right] & 1 \\ -\frac{1}{2}\left[x^{(1)}(2)+x^{(1)}(3)\right] & 1 \\ \cdots & \ldots \\ -\frac{1}{2}\left[x^{(1)}(n-1)+x^{(1)}(n)\right] & 1\end{array}\right]$

(4)Determine equation parameters

Obtaining parameters according to the principle of least squar:

$$
\begin{aligned}
& \hat{A}=\left(B^{T} B\right)^{-1} B^{T} Y_{n}=\left[\begin{array}{l}
\hat{a} \\
\hat{u}
\end{array}\right] . \\
& \hat{c}=\left(x^{(1)}(1)-\frac{\hat{u}}{\hat{a}}\right) \times e^{-\hat{a}}
\end{aligned}
$$

(5)Establish time response function

Through the above calculation,the time response function of the model can be obtained as follows:

$x^{(1)}(t)=\left[x^{(1)}(1)-\frac{\hat{u}}{\hat{a}}\right] e^{-\hat{a} t}+\frac{\hat{u}}{\hat{a}}$

(6)Determine original value

Making inverse accumulated generating on $x^{(1)}(t)$, reducing value can be obtained as follows:

$\hat{x}^{(0)}(t+1)=\hat{x}^{(1)}(t+1)-\hat{x}^{(1)}(t)$

$=\left(1-e^{\hat{a}}\right)\left(x^{(0)}(1)-\frac{\hat{u}}{\hat{a}}\right) \bullet e^{-\hat{a} t}$

\subsection{Model test theory}

Applying a prediction model to a specific object, a series of prediction data can be obtained.In order to know the quality of prediction results ,the prediction data need to be compared to the original data,that is model test.Normally,a good test includes the following aspects[Che-Chiang Hsu,2003]:

(1)Residual analysis

Residual,also known as residual error,is the difference between the actual value and the calculated value of model. 
Pixel residuals :

$\varepsilon^{(0)}(t)=x(0)(t)-\hat{x}(0)(t)(t=1,2, \wedge, n)$

The average residual

$\bar{\varepsilon}=\frac{1}{n} \sum_{k=1}^{n} \varepsilon^{(0)}(t)$

Residual variance:

$S_{1}^{2}=\frac{1}{n} \sum_{t=1}^{n}\left[\varepsilon^{(0)}(t)-\bar{\varepsilon}\right]^{2}$

Pixel relative residuals:

$\phi_{t}=\frac{\varepsilon^{(0)}(t)}{x(0)(t)}$

The average relative residuals:

$\bar{\Phi}=\frac{1}{n} \sum_{i=1}^{n}\left|\phi_{i}\right|$

(2)Fitting accuracy analysis[A. Sfetsos,2004]

Average value of Original data:

$\bar{x}=\frac{1}{n} \sum_{t=1}^{n} x^{(0)}(t)$

Variance of original data:

$S_{2}^{2}=\frac{1}{n} \sum_{t=1}^{n}\left[x^{(0)}(t)-\bar{x}\right]^{2}$

So, Posterior variance ratio:

$C=\frac{S_{1}}{S_{2}}$

Small error probability:

$P=P\left\{\left|\mathcal{E}^{(0)}(t)-\bar{\varepsilon}\right|<0.6745 S_{2}\right\}$

Prediction accuracy can be divided into four grades based on the values of $\mathrm{p}$ and $\mathrm{c}$, the specific division is as follows:

Tab1. Classification of prediction accuracy

\begin{tabular}{ccc}
\hline $\begin{array}{c}\text { Level of Prediction } \\
\text { Accuracy }\end{array}$ & P & C \\
\hline A & $>0.95$ & $<0.35$ \\
B & $>0.8$ & $<0.5$ \\
D & $>0.7$ & $<0.45$ \\
E & $\leqslant 0.7$ & $\geqslant$ \\
& & 0.65 \\
\hline
\end{tabular}

(3) Correlation inspection:

Pixel correlation coefficient:

$\eta_{(k)}=\frac{\min _{j} \min _{l}\left|x_{0}(l)-x_{j}(l)\right|+\lambda \max _{j} \max _{l}\left|x_{0}(l)-x_{j}(l)\right|}{\left|x_{0}(l)-x_{j}(l)\right|+\lambda \max _{j} \max _{l}\left|x_{0}(l)-x_{j}(l)\right|}$

Correlation of $x_{0}$ and $x_{\mathrm{i}}$ :

$$
r_{i}=\frac{1}{n} \sum_{k=1}^{n} \eta_{i}(k)
$$




\section{IMPROVED GM (1,1) MODEL}

When Calculating the parameter $\mathrm{c}$ of differential equations,traditional $\operatorname{GM}(1,1)$ model considers only the $x^{(1)}(1)$ of 1 -AGO,there could be a large error in the calculation process.This paper put forward a new way to Calculate Parameters c,The process is as follows:

(1)Establish the function of residual sum of squares

$$
\left.F(c)=\sum_{t=1}^{n} \hat{x}^{(0)}(t)-x^{(0)}(t)\right)^{2}=\sum_{t=1}^{n}\left(c e^{-a t}\left(1-e^{a}\right)-x^{(0)}(t)\right)^{2}
$$

(2)Determine equation parameter c

In order to obtain best parameter c,the $F(c)$ 'need to be 0 .That is

$$
c=\frac{\sum_{t=1}^{n} x^{(0)}(t) e^{-a t}}{\sum_{t=1}^{n} e^{-2 a t}\left(1-e^{a}\right)}
$$

(3)Establish time response function

Through the above calculation,the time response function of the model can be obtained as follows:

$$
x^{(1)}(t)=\frac{\hat{u}}{\hat{a}}+e^{-\hat{a} t} \frac{\sum_{t=1}^{n} x^{(0)}(t) e^{-\hat{a} t}}{\sum_{t=1}^{n} e^{-2 \hat{a} t}\left(1-e^{\hat{a}}\right)}
$$

(4)Determine original value

Making inverse accumulated generating on $x^{(1)}(t)$, reducing value can be obtained as follows:

$\hat{x}^{(0)}(t+1)=\hat{x}^{(1)}(t+1)-\hat{x}^{(1)}(t)$

$$
=\left(e^{-\hat{a}(t+1)}-e^{-\hat{a} t}\right) \frac{\sum_{t=1}^{n}(0)(t) e^{-\hat{a} t}}{\sum_{t=1}^{n} e^{-2 \hat{a} t}\left(1-e^{a}\right)}
$$

\section{EXAMPLE ANALYSIS}

\subsection{Model prediction}

In order to prove the progressiveness of improved method,this paper takes the power load data of Tianjin from 2001 to 2010 as the research object.

This paper respectively uses traditional GM $(1,1)$ model and improved GM $(1,1)$ model to predict data from 2011 to 2013 according to data from 2001 to 2010.The original data and prediction data shown in Tab.2. 
Tab.2 Original data, prediction data based on traditional GM $(1,1)$ model and improved GM(1,1) model

\begin{tabular}{|c|c|c|c|}
\hline Time & Original data & $\begin{array}{l}\text { Traditional } \\
(1,1) \quad \text { Model }\end{array}$ & $\begin{array}{c}\text { GM Improved } \\
\text { Model }\end{array}$ \\
\hline 2001 & 247.9400 & 247.9400 & 247.9400 \\
\hline 2002 & 274.3900 & 285.3402 & 285.1179 \\
\hline 2003 & 313.0000 & 315.3910 & 315.1452 \\
\hline 2004 & 350.9700 & 348.6065 & 348.3349 \\
\hline 2005 & 384.8400 & 385.3201 & 385.0200 \\
\hline 2006 & 433.6500 & 425.9003 & 425.5686 \\
\hline 2007 & 494.9100 & 470.7542 & 470.3875 \\
\hline 2008 & 515.8800 & 520.3319 & 519.9266 \\
\hline 2009 & 550.1600 & 575.1309 & 574.6829 \\
\hline 2010 & 645.7400 & 635.7011 & 635.2060 \\
\hline 2011 & 695.1500 & 702.6503 & 702.1030 \\
\hline 2012 & 722.4800 & 776.6503 & 776.0453 \\
\hline 2013 & 774.4900 & 858.4436 & 857.7749 \\
\hline
\end{tabular}

\subsection{Model test}

(1)Residual analysis

Residuals of models from 2011to 2013 is shown in Tab.3 .

Tab.3 Residuals of models from 2011to 2013

\begin{tabular}{ccccccc}
\hline $\begin{array}{c}\text { Original } \\
\text { data }\end{array}$ & $\begin{array}{c}\text { Traditional GM }(1,1) \\
\text { value }\end{array}$ & $\begin{array}{c}\text { model } \\
\text { residual }\end{array}$ & $\begin{array}{c}\text { Improved GM(1,1) } \\
\text { residual } \\
(\%)\end{array}$ & $\begin{array}{c}\text { Prediction } \\
\text { value }\end{array}$ & $\begin{array}{c}\text { Absolute } \\
\text { residual }\end{array}$ & $\begin{array}{c}\text { Relative } \\
\text { residual } \\
(\%)\end{array}$ \\
\hline 695.15 & 702.6503 & -7.5003 & -1.0789 & 702.103 & -6.953 & -1.0002 \\
722.48 & 776.6503 & -54.1703 & -7.4978 & 776.0453 & -53.5653 & -7.4141 \\
774.49 & 858.4436 & -83.9536 & -10.8399 & 857.7749 & -83.2849 & -10.7535 \\
\hline
\end{tabular}

The average residual of Improved GM(1,1) model is $6.38927 \%$, and the level of Traditional GM $(1,1)$ model is $6.4722 \%$.It proves that improved prediction model can do improve prediction precision.

(2)Fitting accuracy analysis 4.

Judging from values of $C$ and $P$,the Two models are both in level 'A',the result is shown in Tab.

Tab. 4 Evaluation grade table of Improved GM $(1,1)$ Compared with Original Model

\begin{tabular}{rcc}
\hline Index & $\begin{array}{c}\text { Traditional GM (1,1) } \\
\text { Model }\end{array}$ & $\begin{array}{c}\text { Improved GM(1,1) } \\
\text { Model }\end{array}$ \\
\hline C & $0.1628<0.35$ & $0.1621<0.35$ \\
P & $1>0.95$ & $1>0.95$
\end{tabular}

(3) Correlation inspection:

Level A $\quad$ A

Correlation Degree of improved model and the original data is 0.6457 ,and the Traditional grey prediction model is 0.6459 . Greater degree of similarity exists in the improved model and the original data than the original model. 


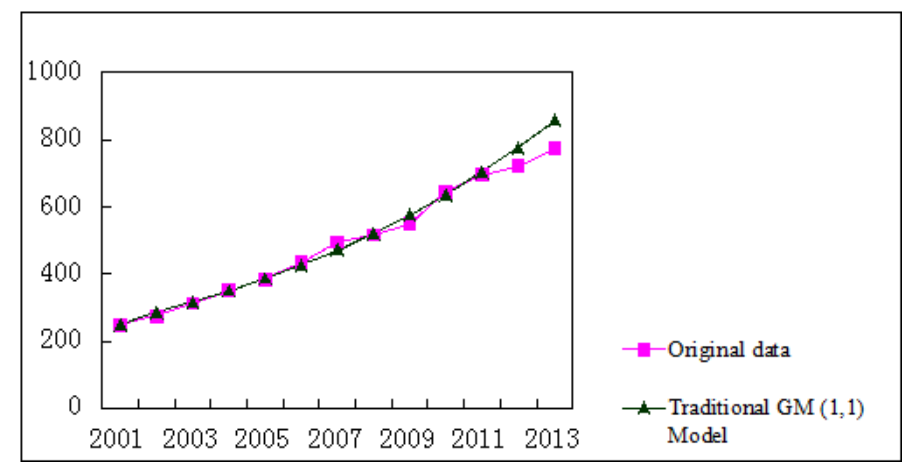

Figure 1. Prediction data based on traditional model.

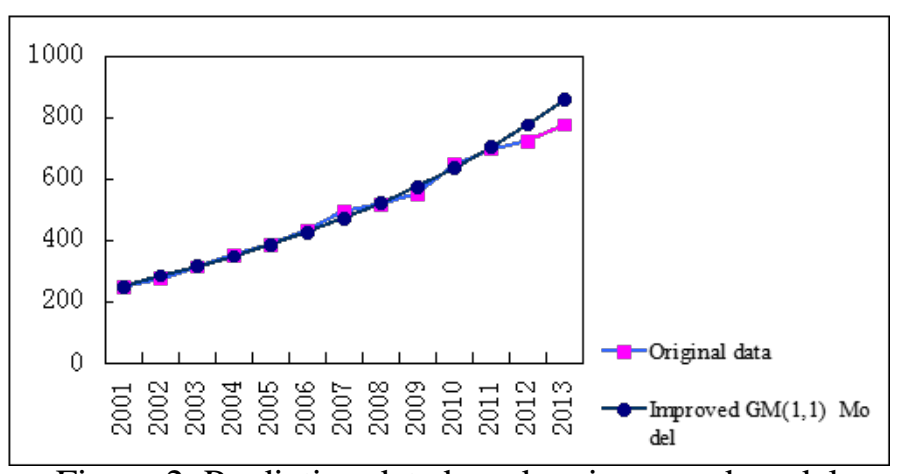

Figure 2. Prediction data based on improved model

\section{CONCLUSION}

Through the above analysis ,This paper gives a new way to calculate the initial value.It compares prediction results between traditional grey prediction model and improved grey prediction model through actual example and proves that improved grey prediction model can improve prediction precision.Model test shows that the improved GM $(1,1)$ model not only retains the advantages of traditional model to calculate simplicity, but also improves the prediction accuracy.

\section{REFERENCES}

[1] Ren Gonghang,et al.2010.Prediction and implementation of the improvement grey model to electrical load .Journal of MachineryDesign\&Manufacture 2:232-234

[2] Deng Julong.2000.Positional target in grey target theory. Journal of The Journal ofGrey System 1: $1-8$

[3] Wang Changjiang.2006.Selection of Smoothing Coefficient via Exponential Smoothing Algorithm.Journal of North University of China(Natural Science Edition) 27(6):58-61

[4] Contreras J, et al. 2003.ARIMA models to predict next-day electricity prices. Journal of IEEE Trans on Power Systems 18(3):1014- 1020.

[5] Che-Chiang Hsu et al.2003.Applications of improved grey prediction model for power demand forecasting. Journal of Energy Conversion and management 44:55-57

[6] Sfetsos et al .2004. Short Term Load Forecasting with a Hybrid Clustering Algorithm and Pattern Recognition. Journal of Engineering Intelligent Systems for Electrical Engineering and Communications 12(1): 13-19 